\title{
IL MINORE VITTIMA DI ABUSI SESSUALI E LE GARANZIE DEL GIUSTO PROCESSO PENALE
}

\author{
Luzi E. \\ eleonora.luzi@uniroma1.it \\ Esposito F. \\ Damato F. \\ Cestari M. \\ Ricci S. \\ Petrone L.
}

Fecha de Recepción: 23 Marzo 2018

Fecha de Admisión: 10 Abril 2018

\section{SOMMARIO}

Nei procedimenti penali aventi ad oggetto l'accertamento della condotta di abuso sessuale a danno di minore, fra le fasi più delicate, vi è quella inerente l'acquisizione delle dichiarazioni della giovane vittima specie se in tenera età.

E' pacifico che l'assunzione della testimonianza di un minore, perché tale, deve avvenire con criteri e modalità che abbiano quale obiettivo principale, quello della tutela del minore stesso.

II sistema penale italiano a tutt'oggi rileva l'assenza di un organico sistema di protezione del minore vittima di abusi; manca una specifica disciplina da parte del legislatore, della testimonianza del soggetto minore di età.

Secondo quanto disposto dall'art. 196, comma 1 e 2 c.p.p. “Ogni persona ha la capacità di testimoniare" tuttavia "qualora, al fine di valutare le dichiarazioni del testimone, sia necessario verificarne l'idoneità fisica 0 mentale a rendere testimonianza, il giudice anche di ufficio può ordinare gli accertamenti opportuni con i mezzi consentiti dalla legge".

Più efficacemente la Carta di Noto all'art. 10 raccomanda per i minori di anni dodici e salvo casi eccezionali "che sia sempre disposta perizia al fine di verificarne la idoneità a testimoniare sui fatti oggetto d'indagine", salvo al giudice valutare l'attendibilità della testimonianza resa.

E' possibile allora per il decidente, accertare la veridicità della condotta abusante dal racconto del minore, prescindendo dall'ausilio del parere tecnico di un perito, ovvero senza aver prima disposto una perizia psicologica sul bambino?

L'orientamento giurisprudenziale prevalente negli ultimi anni, conferma il prevalere del principio cosiddetto della valutazione onnicomprensiva, per cui "in tema di reati sessuali su minori in tenera età, è illegittimo, per violazione del principio della formazione della prova in contraddittorio, il rifiu- 


\section{IL MINORE VITTIMA DI ABUSI SESSUALI E LE GARANZIE DEL GIUSTO PROCESSO PENALE}

to dei giudice di disporre una perizia psicologica, al fine di accertare l'aderenza alla realtà o meno della narrazione dei fatti, in dipendenza di eventuali elaborazioni fantasiose proprie dell'età 0 della struttura personologica del minore"1.

Parole chiave: abusi sessuali collettivi; minore; testimonianza; perizia psicologica; procedimento penale

\section{ABSTRACT}

Child sexual abuse and the guarantees of a fair criminal trial.

Criminal proceedings concerning the assessment of sexual abusive behaviour affecting minors includes, among its most delicate stages, the collection of the young victim's statements, especially if the victims are in their earliest years.

The collection of a minor's testimony must follow rules and procedures whose main goal is to safeguard the minor.

Up to now, Italian Criminal Justice System does not provide an organic system for the young victim's protection; on the part of the legislator, there is a lack of specific rules and regolations for the minor's testimony.

According to Article 196, paragraph 1 and 2 of the Italian Code of Criminal Procedures, "every person is competent to testify," but "in order to evaluate the witness' statement, the judge can order appropriate verifications using all lawful means, even ex officio."

More effectively, Article 10 of the Carta of Noto recommend, for children under 12 and apart from exceptional cases, "that a valuation must always occur in order to verify the victim's fitness to testify about the matters," unless the judge can evaluate the reliability of the testimony.

Therefore, is it possible for the judge to establish the truthfulness of the minor's testimony apart from a technical advice of an expert, that is without making a psychological evaluation of the child first?"

The prevailing jurisprudential orientation in recent years confirms the prevalence of the so-called principle of the all-encompassing evaluation, whereby "on the subject of sexual offenses against young children, it is illegal, for violation of the principle of the formation of evidence in contradictory, the refusal of judge to arrange a psychological examination, in order to ascertain the adherence to the reality or not of the narration of the facts, depending on possible fanciful elaborations of the age or of the personological structure of the minor $2 "$.

Keywords: collettive sexual abuse; minor; testimony; psychological evaluation; criminal proceedings

\section{THE BEST INTERESTS OF THE CHILD E IL DIRITTO ALL'ASCOLTO DEL MINORE}

La rivelazione e l'accertamento di un fatto di abuso sessuale a danno di un minore è un procedimento estremamente complesso, dove ogni singolo atto o operazione deve essere adeguato alle peculiari caratteristiche della giovane vittima di abuso.

La Convenzione di New York sui diritti del fanciullo (Focarelli 2010), all'art. 3, par. 1, disciplina il principio del superiore interesse del minore nelle decisioni che lo riguardano, parimenti la Carta dei diritti fondamentali dell'Unione Europea (2002), all'art. 24, par. 2, statuisce che "in tutti gli atti relativi ai bambini, siano essi compiuti da autorità pubbliche 0 da istituzioni private, l'interesse superiore del bambino deve essere considerato preminente", vale a dire che in tutte le azioni che riguardano minori, intraprese da autorità pubbliche 0 da istituzioni private, la considerazione primaria deve essere la tutela del migliore interesse del bambino, the best interests of the child, quale principio generale del sistema giuridico di tutela del soggetto minore di età. 
Gli strumenti internazionali dedicati al minore, pure informandovisi, non definiscono il principio del superiore interesse del minore, riservando al potere discrezionale dell'interprete, il compito di specificare volta per volta il significato effettivo di tale formula.

Nella prassi giudiziaria, the best interests of the child, trova attuazione nel diritto del minore ad essere ascoltato.

II diritto del minore ad essere ascoltato è confermato in numerose convenzioni di diritto internazionale, in particolare, la Convenzione ONU sui diritti del fanciullo (Théry, 1994), all'art. 12, sottolinea il diritto all'ascolto delle opinioni del minore, affermando che il bambino, ragazzo e adolescente, deve avere la possibilità di essere ascoltato in ogni procedura giudiziaria 0 amministrativa che lo riguarda, sia direttamente sia tramite un rappresentante 0 un organo appropriato ed in maniera compatibile con le regole di procedura della legislazione nazionale.

La libertà di opinione del bambino è tutelata altresì dalla Carta di Nizza (Villani, 2004), il cui art. 24, stabilisce che "I bambini [...] possono esprimere liberamente la propria opinione; questa viene presa in considerazione sulle questioni che li riguardano in funzione della loro età e della loro maturità".

L'ascolto del minore è un vero e proprio diritto riconosciuto al bambino, espressione processuale del principio di preminenza dell'interesse del minore su ogni altro interesse, quale criterio di bilanciamento dei diritti coinvolti e a cui deve essere improntata la definizione della controversia tutte le volte in cui ad essere coinvolti sono uno o più diritti garantiti dall'ordinamento allo stesso minore (Luzi, 2016).

II principio del superiore interesse del minore e il suo diritto di essere ascoltato sono stati recepiti nell'ordinamento italiano con la Legge n. 219 del 10 dicembre 2012 e il successivo Decreto Legislativo n. 154 del 28 dicembre 2013, che introducendo gli artt. 315 bis, 336 bis e 337 octies, cod. civ., hanno confermato la volontà del legislatore italiano di riconoscere centralità al ruolo del minore, tanto nella relazione con i genitori, quanto all'interno del processo, nella definizione delle controversie che lo riguardano

L'art. 315 bis cod. civ., in particolare, al comma 3 stabilisce che "Il figlio minore che abbia compiuto gli anni dodici, e anche di età inferiore ove capace di discernimento, ha diritto di essere ascoltato in tutte le questioni e le procedure che lo riguardano".

L'art. 336 bis cod. civ., specifica che il minore sia ascoltato dal giudice nell'ambito dei procedimenti nei quali devono essere adottati provvedimenti che lo riguardino, salvo il caso in cui l'ascolto sia in contrasto con il suo interesse 0 manifestamente superfluo, di cui deve darsi atto mediante provvedimento adeguatamente motivato, dal momento che "costituisce violazione del principio del contraddittorio e dei principi del giusto processo il mancato ascolto che non sia sorretto da espressa motivazione sull'assenza di discernimento che ne può giustificare l'omissione"3.

L'art. 337 octies cod. civ., conferma, tra i poteri che il giudice può esercitare prima dell'emanazione, anche in via provvisoria, dei provvedimenti riguardo ai figli, di cui all'art. 337 ter cod. civ, quello di disporre l'audizione di questi ultimi.

Il legislatore riconosce, pertanto, il diritto del bambino di potersi esprimere, di poter narrare, raccontare e far sentire la propria voce in tutte le procedure, siano esse giudiziarie 0 amministrative, che lo interessino "salvo che l'ascolto possa essere in contrasto con il suo superiore interesse"4.

Si comprende come anche all'interno del sistema giuridico italiano, il legislatore abbia inteso delineare l'audizione dei minori di età, quale adempimento necessario, in tutte le procedure giudiziarie che li riguardino, costituendo "una modalità, tra le più rilevanti, di riconoscimento del diritto fondamentale del minore ad essere informato ed esprimere la propria opinione e le proprie opzioni" nonché "elemento di primaria importanza nella valutazione del suo interesse" 5 . 


\section{ABUSO SESSUALE SU MINORE: L'IMPORTANZA DI UNA DEFINIZIONE UNIVOCA}

L'audizione testimoniale di un minore, è un'esigenza che può manifestarsi in ambiti e con finalità diverse.

Nei procedimenti penali aventi ad oggetto l'accertamento della condotta di abuso sessuale a danno di minore, l'audizione del minore vittima del presunto fatto di abuso, costituisce la regola.

La rivelazione 0 la rilevazione, a seconda che il presunto fatto di abuso emerga in forma esplicita ovvero attraverso indicatori comportamentali, costituiscono operazioni estremamente difficili, soprattutto perché sussiste tra gli operatori molta confusione, dovuta per lo più alla mancanza di una definizione condivisa, su cosa debba intendersi per abuso sessuale (Scardaccione, 2002; Finkelhor, 1986).

Si sottolinea insistentemente, che presupposto della corretta comunicazione tra i diversi professionisti che operano nelle aree socio-sanitaria e giuridica è stabilire una definizione chiara, univoca e condivisa di abuso sessuale su minore.

Così si è constatato che "nella quasi totalità dei casi l'abuso in danno del minore, ancor prima di essere fisico, psicologico o sessuale è caratterizzato da una situazione di abuso di posizione dominante, nell'ambito di relazioni interne al gruppo, sia esso famigliare o no, gravemente distorte.

La posizione di superiorità e di potere sul minore, che si trova invece in una posizione di dipendenza e di soggezione rispetto ad essa, rappresenta infatti la condizione facilitante che consente all'aggressore di costringere, implicitamente 0 esplicitamente, il minore a subire l'abuso.

La maggior parte degli autori (Kempe, 1962; Montecchi, 1994; CISMAi, 1998; WHO, 1999; WHO, 2003) è concorde nel definire l'abuso sessuale sul minore come il coinvolgimento di un soggetto di minore età in manifestazioni ed atti sessuali che egli non comprende appieno e per i quali, non avendo raggiunto piena maturità psicologica, non è in grado di esprimere consapevole consenso.

Nella definizione proposta dal Consiglio d'Europa durante il IV Seminario Criminologico che si è svolto a Strasburgo nel 1978, per abuso sessuale di un minore deve intendersi "ogni atto o carenza che turbi gravemente i bambini o le bambine, che attenta alla loro integrità corporea, al loro sviluppo psico-fisico, affettivo, intellettivo e morale, le cui manifestazioni sono la trascuratezza e/o le lesioni di ordine fisico e/o psichico e/o sessuale da parte di un familiare o di un terzo, ed ogni atto sessuale imposto al bambino non rispettando il suo libero consenso".

Anche nel codice penale italiano, nel dispositivo dell'art. 609 bis, introdotto con la Legge n. 66 del 15 febbraio 1996, si parla genericamente di atti sessuali, con una definizione onnicomprensiva che vi ricomprende qualunque atto espressione di desiderio sessuale, che può interessare zone erogene differenti, che sia idoneo ad invadere la sfera sessuale del soggetto passivo mediante costringi mento, che pertanto violi la libera autodeterminazione nel campo della sessualità 6

Presupposto necessario di tale delitto è che l'atto sessuale sia associato al costringimento del soggetto passivo.

Esiste tuttavia, un'importante differenza tra la definizione clinica e la definizione giuridica di abuso sessuale.

II concetto clinico di abuso sessuale elaborato dalla letteratura scientifica risulta infatti più ampio rispetto alla condotta penalmente rilevante sul piano giudiziario.

La descrizione della fattispecie di reato anche nella Legge $n$. 66 del 1996 richiama la costrizione della vittima a "compiere o subire atti sessuali" senza rispettare il suo libero consenso.

Nella definizione clinica, oggetto di tutela però, è il corretto sviluppo della identità sessuale e della personalità del minore, il quale può essere danneggiato da qualunque atto sessuale, di qualsivoglia natura, chiunque sia il soggetto agente, e altresì da tutte quelle situazioni, che pur non costi- 
tuendo veri e propri "atti", non differiscono affatto da quelle codificate invece come violenza sessuale, in quanto le ripercussioni in termini di danno psichico potrebbero essere le medesime.

La Legge n. 66 del 1996, invece, appresta tutela alla libertà di autodeterminazione della persona nella sfera sessuale, la lesione della quale diventa presupposto essenziale alla configurabilità del reato di violenza sessuale ex art 609 bis cod. pen..

Nella norma penale, la condizione di minore età non soltanto fa presumere la violenza dell'atto sessuale indipendentemente dal consenso espresso dalla vittima, bensì anche lo stato d'inferiorità fisica e psichica propria del minore e dunque l'impossibilità di questo di esprimere un consapevole consenso.

L'art. 609 quater, sottolineando al $2^{\circ}$ comma, che la differenza di età tra soggetti minorenni, affinché possa non configurarsi il reato di abuso sessuale, debba essere al massimo di 3 anni, e che il minore abbia almeno 13 anni, interviene a delimitare la portata applicativa della norma incriminatrice, riconoscendo altresì il diritto del minore di esprimere la propria sessualità, senza alcuna penalizzazione.

Se si considera che la stessa Dichiarazione di consenso in tema di abuso sessuale all'infanzia, approvata a Roma nel 1998, definisce l'abuso sessuale come "il coinvolgimento di un minore da parte di un partner preminente in attività sessuale anche non caratterizzata da violenza esplicita [...] fenomeno diffuso, che si configura sempre e comunque come un attacco confusivo e destabilizzante alla personalità del minore e al suo percorso evolutivo" si ha che nell'area della definizione clinica di abuso su minore, l'intervento di tutela della personalità del minore destabilizzato, viene attivato indipendentemente dalle modalità dell'atto sessuale compiuto e dall'età del minore; ai fini dell'applicabilità della norma penale invece, per poter affermare e poter valutare il grado di responsabilità del soggetto agente, acquistano rilievo non soltanto quegli atti che sono espressione di desiderio sessuale e sono al contempo idonei ad invadere la sfera sessuale del soggetto abusato, bensì pure le modalità dell'atto sessuale e l'età della vittima .

\section{LA QUESTIONE DEI COSIDDETTI RIVELATORI O “INDICATORI” DI UNA VITTIMIZZAZIONE}

In sede di accertamento di un presunto caso di abuso sessuale su minorenne, alle difficoltà definitorie accennate, si affiancano difficoltà di natura e origine diagnostica.

Sul tema, la Carta di Noto IV con le Linee guida per l'esame del minore in caso di abuso sessuale, nel recente aggiornamento del 14 ottobre 2017, si è espressa affermando che "Non esistono segnali psicologici, emotivi e comportamentali validamente assumibili come rivelatori o indicatori di una vittimizzazione. Non è scientificamente fondato identificare quadri clinici riconducibili ad una specifica esperienza di abuso, né ritenere alcun sintomo prova di essi. Parimenti, l'assenza di sintomatologia psicologica, emotiva e comportamentale in capo al minore non può escluderli7".

Tale considerazione, si ricollega a quella corrente di pensiero secondo la quale non esistono indicatori specifici che possano escludere o confermare in modo certo l'avvenuto abuso sessuale sul minore, sempre che, come alcuni sottolineano "non si concentrino nel minore molteplici indicatori ovvero indici sia fisici che psicologici di abuso".

Occorre per questo motivo evitare il "rischio di vedere una correlazione illusoria tra causa supposta (abuso sessuale) e conseguenze (indicatori), dove questa non c'è".

L'equivocità può infatti riguardare gli indicatori fisici, ma anche quelli psicologici o comportamentali.

L'assenza di riscontro di segni patognomonici sul corpo della vittima, fa si che la stessa indagine medico legale, che pure rappresenta il mezzo di prova di maggiore utilizzo insieme alla audizione della vittima (Gelpi, 2002), non sempre produca risultati significativi ai fini del giudizio finale. 
Durante gli accertamenti, assai frequentemente sono rilevabili segni aspecifici, quelli, ad esempio, relativi alla psiche del minore, come i fenomeni di autolesionismo o la depressione, che non costituiscono di per sé sintomi di abuso sessuale.

Più spesso accade che la denuncia di reato e di conseguenza l'esame obiettivo siano tardivi, così che gli eventuali segni di abuso risultano essere non più visibili (McCann, Voris, Simon, 1992, Del Vecchio,1998, WHO, 2003).

Per di più, occorre tener presente che non sempre il bambino abusato assume comportamenti sintomatici manifesti, e anche quando ciò si verifica, l'atteggiamento sintomatico o "segnale", può non essere immediatamente manifestato.

L'assenza dunque di indicatori fisici, psicologici o comportamentali specifici, spiega il rilievo dato alla testimonianza del minore vittima di abuso, ma anche alle modalità di acquisizione e a quelle di valutazione della prova testimoniale (Agnoli \& Ghetti, 1995).

\section{L'AUDIZIONE DEL MINORE VITTIMA DI ABUSI SESSUALI: GARANZIE E LIMITI}

Nel processo penale per l'accertamento di un fatto di abuso sessuale a danno di minore, fra le fasi più delicate, vi è quella inerente l'acquisizione delle dichiarazioni della giovane vittima specie se in tenera età.

La testimonianza, ovvero il resoconto di un bambino su un fatto da lui osservato, in quanto è rivolta all'accertamento dei fatti, si differenzia dal mero ascolto del minore, che è lo strumento che gli consente di partecipare alle decisioni che lo riguardano ed è rivolto, non all'accertamento dei fatti oggetto di indagine, bensì alla cognizione delle sue opinioni, dei suoi giudizi e dei suoi pensieri.

Si è osservato che "se la notitia criminis può scaturire da una fonte diversa dal racconto del minore, è difficile immaginare un processo penale che possa prescinderne completamente".

E' pacifico che l'assunzione della testimonianza di un minore, perché tale, deve avvenire con criteri e modalità che abbiano quale obiettivo principale, quello della tutela del minore stesso.

II sistema penale italiano a tutt'oggi rileva l'assenza di un organico sistema di protezione del minore vittima di abusi; manca una specifica disciplina da parte del legislatore, della testimonianza del soggetto minore di età.

Secondo quanto disposto dall'art. 196, comma 1 e 2 cod. proc. pen. "Ogni persona ha la capacità di testimoniare" tuttavia "qualora, al fine di valutare le dichiarazioni del testimone, sia necessario verificarne l'idoneità fisica o mentale a rendere testimonianza, il giudice anche di ufficio può ordinare gli accertamenti opportuni con i mezzi consentiti dalla legge".

La minore età dell'autore della testimonianza, quindi, non incide sulla sua capacità di testimoniare, bensì, piuttosto sulla valutazione della testimonianza e, cioè, sulla sua attendibilità.

Si comprende perciò che la capacità di testimoniare non va affatto confusa con l'attendibilità del teste, in quanto la prima può essere valutata dall'esperto nominato, la seconda rimane di competenza esclusiva del giudice.

La già citata Carta di Noto, sottolinea che "L'idoneità a rendere testimonianza sulla quale l'esperto è chiamato ad esprimersi comprende capacità generiche e specifiche. Le prime riguardano funzioni cognitive quali la memoria, l'attenzione, le capacità di comprensione e di espressione linguistica, la capacità di individuare la fonte delle informazioni, le capacità di discriminare realtà e fantasia, il verosimile dal non verosimile, ecc., nonché il livello di suggestionabilità e di maturità psicoaffettiva. Le capacità specifiche riguardano l'abilità del minore di organizzare e riferire il ricordo in relazione alla complessità esperienziale di quello che si suppone essere avvenuto e l'eventuale presenza di influenze suggestive, interne 0 esterne (derivanti dall'interazione con adulti o con coetanei) che possano avere interferito nel racconto" 8 . 
Più efficacemente, la Carta di Noto raccomanda, per i minori di anni dodici e salvo casi eccezionali, "che sia sempre disposta perizia al fine di verificarne la idoneità a testimoniare sui fatti oggetto d'indagine"9, salvo al giudice valutare la veridicità della testimonianza resa.

Con riferimento alla raccolta delle audizioni, la Carta di Noto sancisce che il minore va sentito in contraddittorio il prima possibile, avvalendosi delle metodiche basate sulle indicazioni della letteratura scientifica accreditata, tenendo presente che "Le audizioni effettuate o ripetute ad una considerevole distanza temporale vanno valutate con grande cautela a causa della condizione psicologica mutata rispetto all'epoca dei fatti e dei potenziali fattori di inquinamento del ricordo" 10 .

Vero è tuttavia, che le raccomandazioni contenute nelle linee guida di cui si è detto, non sempre trovano puntuale riscontro nella giurisprudenza di legittimità che, in diverse pronunce, non proprio Iontane, riconosce ad esse natura di "suggerimenti volti a garantire meglio l'attendibilità delle dichiarazioni del minore e la protezione psicologica dello stesso", negandovi pertanto efficacia vincolante ${ }^{11}$.

D'altronde, per quanto riguarda la possibilità per il decidente, di accertare la veridicità del fatto di abuso dal mero racconto del minore, prescindendo cioè, dall'ausilio del parere tecnico di un perito, ovvero senza aver prima disposto una perizia psicologica sul bambino, il dispositivo di cui all'art. 498 cod. proc. pen., al comma 4, prevede che l'organo giudicante possa avvalersi dell'ausilio di un familiare del minore 0 di un esperto in psicologia infantile.

E' solo di recente che la giurisprudenza sembra muoversi nella direzione che accoglie il principio della cosiddetta valutazione onnicomprensiva affermando che "in tema di reati sessuali su minori in tenera età, è illegittimo, per violazione del principio della formazione della prova in contraddittorio, il rifiuto dei giudice di disporre una perizia psicologica, al fine di accertare l'aderenza alla realtà 0 meno della narrazione dei fatti, in dipendenza di eventuali elaborazioni fantasiose proprie dell'età 0 della struttura personologica del minore"12.

\section{BIBLIOGRAFIA}

Agnoli, F., \& Ghetti, S. (1995). Testimonianza infantile e abuso sessuale. Età evolutiva, 52, 66-75.

CISMAI (Coordinamento Italiano dei Servizi contro il Maltrattamento e l'Abuso all'Infanzia)1998 aggiornato nel 2001: "La Dichiarazione di Consenso in tema di abuso sessuale all'infanzia"

Europea, U. (2000). Carta dei diritti fondamentali dell'Unione Europea. www. europarl. europa. eu/charter/pdf/text_it. Pdf

Finkelhor, D., \& Araji, S. (1986). A sourcebook on child sexual abuse. Sage.

Focarelli, C. (2010). La Convenzione di New York sui diritti del fanciullo e il concetto di” best interests of the child". Rivista di diritto internazionale, 93(4), 981-993.

Kempe, R. S., \& Kempe, C. H. (1989). Le violenze sul bambino. Sovera multimedia.

Luzi E, Centola C, Crusco M. Scambio di embrioni. Genitorialità e status filiationis: tra verità genetica e appartenenza biologica. BioLaw J 2016;2(7):289-300

Montecchi, F. (1994). Gli abusi all'infanzia: dalla ricerca all'intervento clinico. La Nuova Italia Scientifica.

Scardaccione G. La tematica dell'abuso sessuale e i principi dell'intervento, Corso di formazione per ausiliari nella testimonianza dei minori, Roma, 2002.

Théry, I. (1991). La convenzione ONU sui diritti del bambino: nascita di una nuova ideologia. ITALIA. Ministero Dell'Interno. Politiche sociali per l'infanzia e l'adolescenza. Milano: Unicopli.

Villani, U. (2004). I diritti fondamentali tra Carta di Nizza, Convenzione europea dei diriti dell'uomo e progetto di Costituzione europea. I/ Diritto dell'Unione europea, 9(1), 73-116.

World Health Organization. (1999). Report of the Consultation on Child Abuse Prevention, 29-31 March 1999, WHO, Geneva. 


\section{IL MINORE VITTIMA DI ABUSI SESSUALI E LE GARANZIE DEL GIUSTO PROCESSO PENALE}

World Health Organization. (2003). Guidelines for medico-legal care of victims of sexual violence.

1 (Cass. Pen., Sez. III, 13 ottobre 2016, n. 43245)

2 (Cass. Pen., Sez. III, 13 ottobre 2016, n. 43245)

3 (Cass. Civ., SS.UU., 21 ottobre 2009, n. 22238)

4 (Cfr. Cass. Civ., Sez. I, 2 agosto 2013, n. 18538)

5 (Cfr. Cass. Civ., Sez. I, 26 marzo 2015, n. 6129)

6 (Cfr. Cass. Pen., Sez. III, 13 giugno 2017, n. 29235).

7 (Cfr. punto 18 della Carta di Noto IV Linee guida per l'esame del minore 14 ottobre 2017)

8 (Cfr. punto 13 della Carta di Noto IV Linee guida per l'esame del minore)

9 (Cfr. punto 10 della Carta di Noto IV Linee guida per l'esame del minore)

10 (Cfr. punto 2 della Carta di Noto IV Linee guida per l'esame del minore)

11 (Cfr. Cass. Pen. Sez. III 16 ottobre 2014, n. 5754).

12 (Cass. Pen., Sez. III, 13 ottobre 2016, n. 43245) 